XXIV.-On Fagnani's Theorem. By H. F. TALBot.

(Read 20th April 1863.)

Before proceeding to the subject of this paper, I wish to advert to the following well-known theorem.

Let $\mathrm{ABC}$ be any triangle with its inscribed and circumscribed circles. Then if we take any other point $\mathrm{D}$ in the exterior circle, and draw the tangents $\mathrm{DE}, \mathrm{EF}, \mathrm{FD}$, the last tangent will come again to the original point $\mathrm{D}$. A similar theorem is true of a polygon of any number of sides which is both inscribed in, and circumscribed to, a circle. Also if ellipses are substituted for circles. Of these theorems I remember to have seen a demonstration founded on the theory of elliptic integrals. But this appears to me to be a great waste of analytic power; for the theorem really results from first principles, as I think will be manifest from the following considerations.

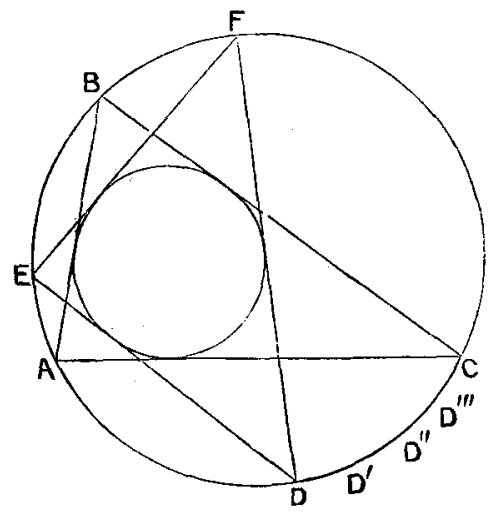

Fig. 1.

I shall take the simplest case, that of two circles inscribed in, and circumscribing, a triangle, because the reasoning is similar in the more complicated cases.

Lemma 1 . If there are two circles, one within the other, and if $\mathrm{AB}, \mathrm{CD}$, are two chords of the outer circle, touching the inner circle, then if $\mathrm{C}$ lies between $\mathrm{A}$ and $\mathrm{B}, \mathrm{D}$ does not.

For it is plain that a line drawn from $C$ to any other point in the arc $\mathrm{AB}$, would not touch the inner circle, which is contrary to the hypothesis.

If a circle lies anyhow within another, and tangents

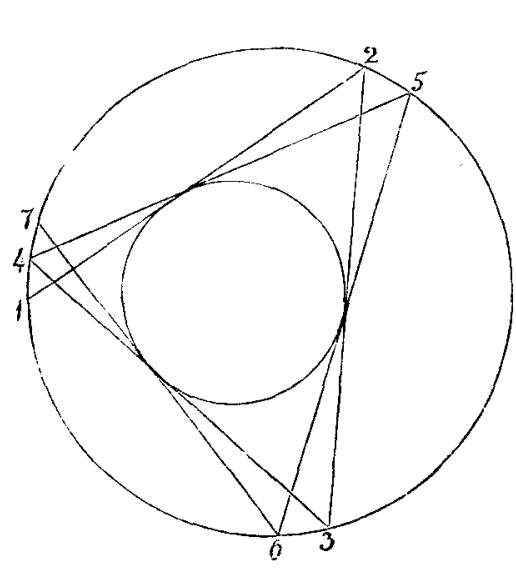

Fig. 3. $1,2-2,3-3,4$, be drawn,

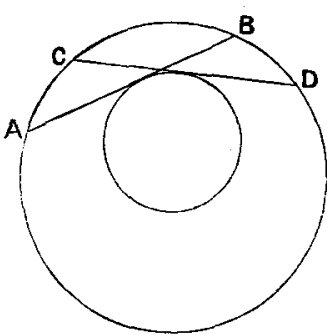

Fig. 2. then, generally speaking, the points 1 and 4 do not coincide. The arc 1, 4, may be called the deviation, and if more tangents $4,5-5,6-6,7$, be drawn, the $\operatorname{arc} 4,7$, is the second deviation.

Lemma 2. The successive deviations are constantly in the same direction, and consequently the total deviation increases till at length it surpasses any given arc of the circle.

Demonstration. Since the point 4 (as represented in fig. 3) lies between 1 and 2, the point 5 , at the other end of the tangent, lies between 2 and 3 (by the first lemma), and for the same reason the point 6 lies between 3 and 4 . vOL. XXIII. PART II. 
Consequently, 7 lies between 4 and 5 ; that is, the deviations 1,4 , and 4,7 , are in the same direction. And the total deviation tends to increase without limit, because it is plain, from the nature of the problem, that the deviations are quantities of the same order (though sometimes larger, sometimes smaller.)

Theorem I. (see fig. 1.) If a triangle $A B C$ is inscribed in one circle and circumscribed about another, then if any point $\mathrm{D}$ be taken in the outer circle, and three tangents, $\mathrm{DE}, \mathrm{EF}, \mathrm{FD}$, be drawn, the extremity of the last will coincide with the original point $\mathrm{D}$.

For if not, let its extremity be $\mathrm{D}^{\prime}$. It is immaterial on which side of $\mathrm{D}$ we suppose $\mathrm{D}^{\prime}$ to lie. Let it be between $\mathrm{D}$ and $\mathrm{C}$. Then calling $\mathrm{D}$ the origin, and $\mathrm{D}^{\prime}$ the terminus, of the three tangents, it follows from lemma 1 , that both $\mathrm{D}$ and $D^{\prime}$ lie between $A$ and $C$. For however near to $C$ we take an origin, the terminus and origin cannot lie on opposite sides of $\mathrm{C}$. Now to return to the case represented in our figure. We have supposed $\mathrm{D}$ the origin, $\mathrm{D}^{\prime}$ the terminus. Take $D^{\prime}$ as a new origin, and $D^{\prime \prime}$ will be the new terminus. Continue the process, and successive termini $\mathrm{D}^{\prime \prime \prime}, \mathrm{D}^{\prime \prime \prime \prime}$, \&c., will be obtained. All the deviations $\mathrm{DD}^{\prime}, \mathrm{D}^{\prime} \mathrm{D}^{\prime \prime}$, \&c., are in the same direction, and the total deviation ultimately surpasses any given arc, and therefore surpasses the arc DC (by lemma 2). But this result involves an absurdity, since it implies that a certain origin $\mathrm{D}_{n}$ and terminus $\mathrm{D}_{n+1}$ are on opposite sides of $\mathrm{C}$. Therefore the supposition from which we started, viz., that $\mathrm{D}$ and $\mathrm{D}^{\prime}$ are different points, leads to a false conclusion. Consequently those points are the same. Q.E.D.

Moreover, the theorem is true when two ellipses, one lying within the other, are substituted for circles. For, by SALmon's Conic Sections, p. 308, two ellipses can be so projected that both will be circles, and of course the projection of the triangle inscribed to the one and circumscribed to the other ellipse, will be a triangle inscribed and circumscribed to the two circles. Such a triangle has the property (as we have just shown) of remaining always closed or complete, however we vary the point of origin, which may be taken in any part of the outer circle.

And therefore all triangles inscribed and circumscribed to the two given ellipses are closed and complete. For otherwise their projections could not be so.

\section{Theorem on the Ellipse.}

In SALMon's Conic Sections, p. 297, we find the following beautiful theorem, due to Dr Graves.

If two ellipses have the same centre and focus, and a pair of tangents be drawn from any point of the outer to the inner ellipse, the difference between the sum of the tangents and the intercepted elliptic arc is a constant quantity. 
From this theorem a very remarkable consequence may be drawn. Let $A B C$, DEF, be the two ellipses having same centre $O$ and foci $S, H$. Moreover, let the axis major of one bear such a proportion to that of the other, that if two chords, $\mathrm{AB}, \mathrm{BC}$, be drawn in the outer ellipse touching the inner one at $\mathrm{E}$ and $\mathrm{F}$, the third chord $\mathrm{CA}$ shall also touch it at $D$. Then by Graves's theorem $\mathrm{BE}+\mathrm{BF}-$ arc $\mathrm{EF}=\mathrm{C}$, a constant. Also $\mathrm{CF}+\mathrm{CD}-\operatorname{arc} \mathrm{FD}=\mathrm{C}$, and $\mathrm{AD}+$ $\mathrm{AE}-\operatorname{arc} \mathrm{DE}=\mathrm{C}$. Therefore adding these equations together, we find that the periphery of the triangle $\mathrm{ABC}$, minus the periphery of the ellipse $=3 \mathrm{C}$. Now, by the preceding theorem I., we know that if, instead of $A$, we take any other point $\mathbf{A}^{\prime}$ of the outer

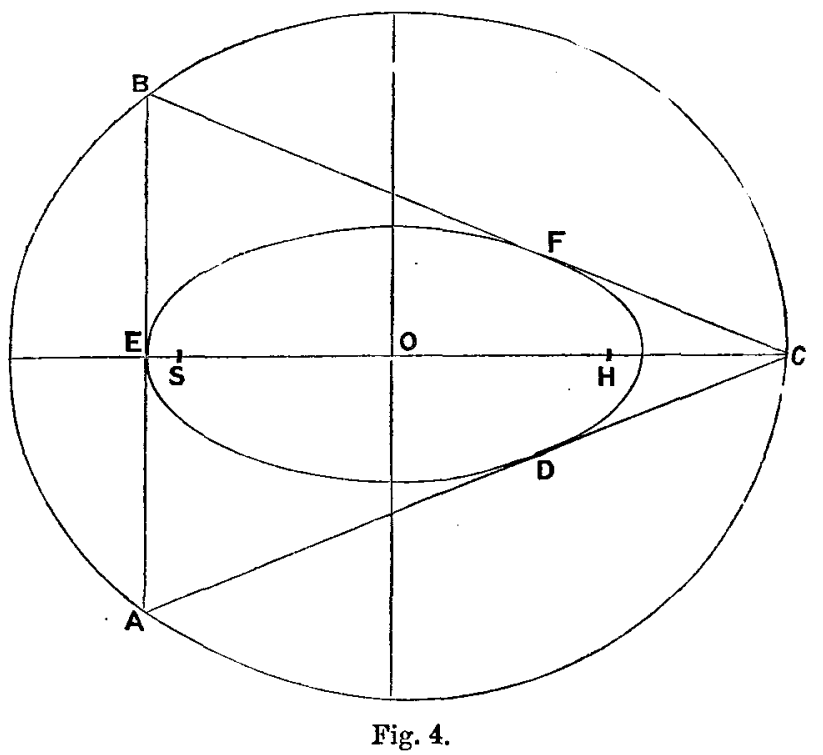
ellipse as the origin, and draw tangents $A^{\prime} B^{\prime}, B^{\prime} C^{\prime}, C^{\prime} A^{\prime}$, from it to the inner ellipse, they will form a closed triangle. Its periphery, minus that of the ellipse, will $=3 \mathrm{C}$, by the same reasoning as before. Therefore the peripheries of the two triangles are equal. But the same reasoning applies to a closed polygon of any number of sides. Whence the following theorem :- "If two ellipses, with same centre and foci are such that a polygon can be inscribed to the one and circumscribed to the other, the periphery of the polygon is constant, whatever point of the outer ellipse be assumed as its origin."

After I had arrived at this theorem, I found that M. Chasles had given one nearly resembling it (Comptes Rendus, 1843, p. 838), with the additional remark, that since every two consecutive sides of the polygon are equally inclined to the periphery of the outer ellipse, a ray of light might describe such a polygon, by successive reflections at the periphery; and therefore it would always be a tangent to an inner confocal ellipse. But I think this remark may be rendered yet more striking by considering the cases in which the polygon is not closed. If this occurs when the origin is any point $A$, it will also occur when any other point is assumed as the origin. The path of the ray of light under these circumstances would be constantly varying, but always tangent to the inner ellipse, so that ultimately, after an infinite number of reflexions, the ray will have passed

* It is unnecessary to say with Chasces that they must be polygons of the same number of sides, for they cannot be otherwise. M. Chasses, in his paper here referred to, gives no demonstrations. I am not aware whether they were subsequently published. 
over every part of the space intercepted between the ellipses, leaving untouched the whole of the interior ellipse.

\section{Fagnani's Theorem.}

Let us now consider MaCCullagh's Theorem, given by SALMON, p. 297.

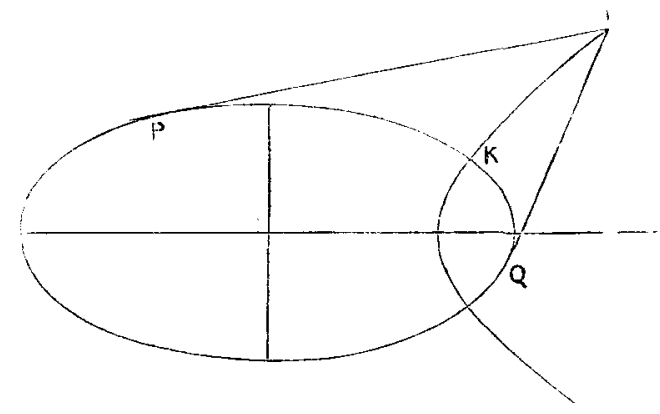

Fig. 5.

If any ellipse is cut by a hyperbola, having same centre and focus in the point $K$, and from any point $T$ of the hyperbola a pair of tangents $\mathrm{TP}, \mathrm{TQ}$, is drawn to the ellipse, the difference of the tangents equals the difference of the arcs $\mathrm{PK}, \mathrm{QK}$.

As an example of this, let tangents be drawn at the extremities of the major and minor axes, meeting in $\mathrm{T}$, and let a hyperbola, having same centre and focus, be so drawn as to pass through $T$, and

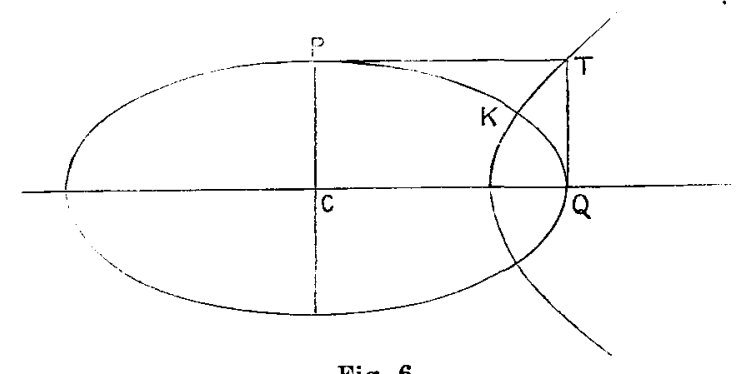

Fig. 6. cut the ellipse in $\mathrm{K}$. Then the elliptic quadrant $P Q$ is so divided in $K$, that $\mathrm{PK}-\mathrm{KQ}=\mathrm{PT}-\mathrm{TQ}=a-b$, or the difference of the semiaxes of the ellipse, which is Fagnani's theorem (SaLmon, p. 298).

Now, let the point $T$ advance continually along the hyperbola, and the intercepted arc will of course always be divided at the same point $\mathrm{K}$. Ulti-

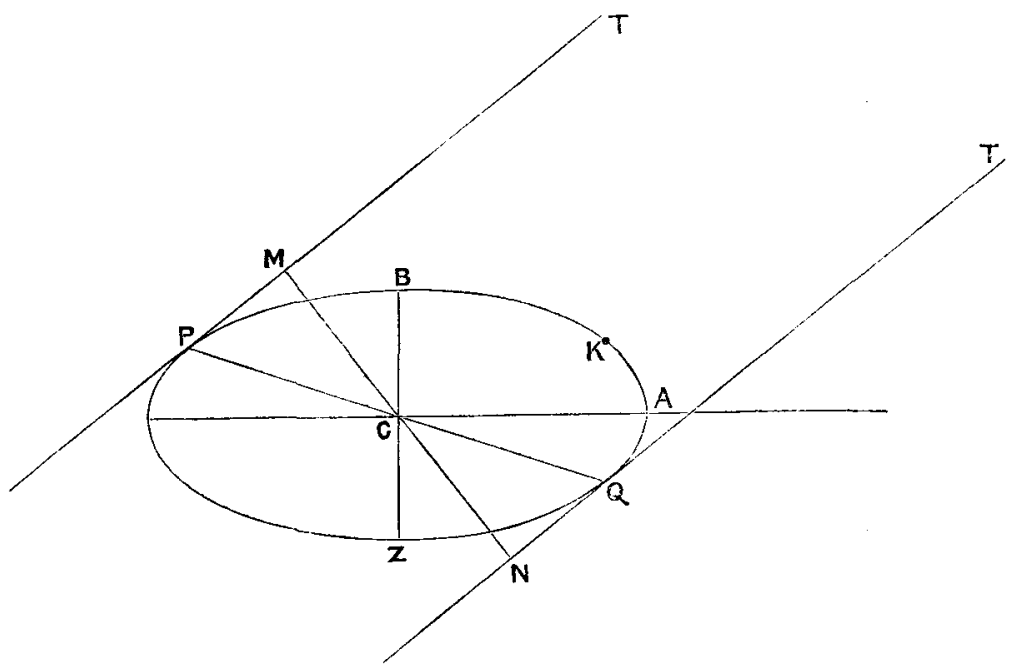

Fig. 7.

mately, the point $\mathrm{T}$ may be supposed to attain the asymptote at an infinite distance. The two tangents TP, TQ, are then parallel to the asymptote. Their 
difference is equal to the difference of their extremities which touch the ellipse, and are cut off by any line at right angles to them. Call this difference D. The intercepted arc is then a seni-ellipse, because, when tangents are parallel, the line joining the points of contact passes through the centre.

Now we have, $\mathrm{PK}-\mathrm{QK}=\mathrm{PT}-\mathrm{QT}=\mathrm{D}$, and $\mathrm{BK}-\mathrm{AK}=a-b$; therefore, by subtraction, $\mathrm{PB}-\mathrm{QA}=\mathrm{D}-(a-b)$. But the arc $\mathrm{PB}=\mathrm{QZ}, \therefore \mathrm{QZ}-\mathrm{QA}=\mathrm{D}-(a-b)$. But ZA is a quadrant of the ellipse, therefore this quadrant is so divided in the point $\mathrm{Q}$, that the difference of $\mathrm{QZ}$ and $\mathrm{QA}$ is a known straight line $=\mathrm{D}-(a-b)$.

Now, it is by no means obvious, whether or not we have thus obtained a division of the elliptic quadrant different from the one we first obtained. This point can only be settled by a rigorous demonstration; the result of which gives this curious theorem $\mathrm{D}=2(a-b)$. From which we see that $\mathrm{QZ}-\mathrm{QA}=a-b=\mathrm{BK}$ $-\mathrm{KA}$, so that the elliptic quadrants $\mathrm{BA}$ and $\mathrm{ZA}$ are divided at corresponding points $K$ and $Q$, and the arc $A K=A Q$. And since the tangent at $Q$ or $Q T$ is parallel to the asymptote, it follows, by parity of reasoning, that the tangent at $\mathrm{K}$ is parallel to the other asymptote. Moreover, if we draw MCN through the centre, at right angles to $P T$ and $Q T$, it is plain that $D=P M+Q N$, or since these lines are equal, $2(a-b)=\mathrm{D}=\mathrm{QQN} \therefore \mathrm{QN}=a-b$, hence the point of division $\mathrm{Q}$ is such, that the perpendicular let fall from the centre on the tangent at $Q$, cuts off from it a portion $\mathrm{QN}=a-b$.

It remains, therefore, to demonstrate $a$ priori the theorem we have just indicated, viz., that $\mathrm{QN}=a-b$, whence the other properties mentioned will follow. We shall, at the same time, obtain the demonstration of many other theorems.

Let CA, CB, be the semi-axes of an ellipse, denoted by $a, b$. Complete the rectangle $B C A D$. Let $A, B$, be the semi-axes of a hyperbola, having same centre and focus, and so drawn as to pass through the point $D$, whose coordinates are $a, b$. Then, according to Salmon (Conic Sections, p. 298), the co-ordinates of the point $P$, which is the

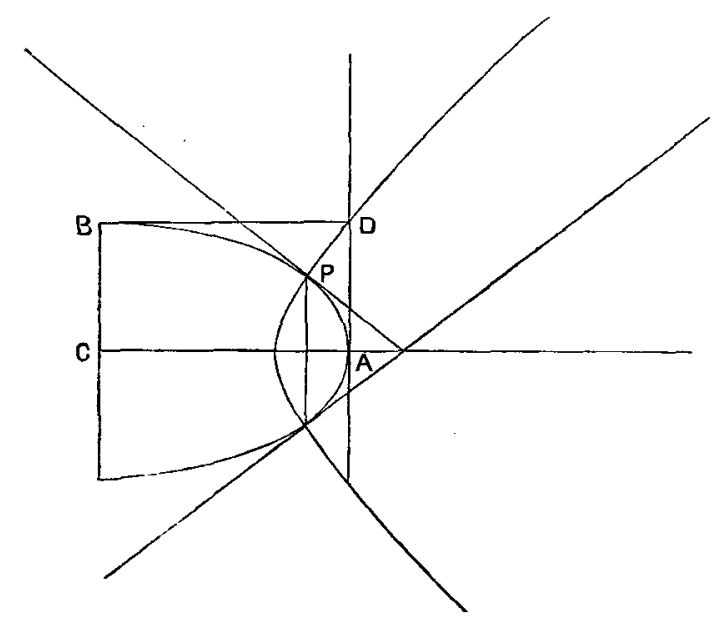

Fig. 8. intersection of the two curves, are, $x^{2}=\frac{a^{3}}{a+b}, y^{2}=\frac{b^{3}}{a+b}$.

We shall reverse this order of reasoning, and suppose a hyperbola drawn with centre $\mathrm{C}$, axis in the line $\mathrm{CA}$, and passing through the points $\mathrm{D}$ and $\mathrm{P}$, and then show that such a hyperbola has the same focus with the ellipse. In the first place, then, the point whose co-ordinates are $x^{2}=\frac{a^{3}}{a+b}, y^{2}=\frac{b^{3}}{a+b}$ lies in the ellipse, 
for those co-ordinates verify the equation to the ellipse $\frac{x^{2}}{a^{2}}+\frac{y^{2}}{b^{2}}=1$. The substitution gives $\frac{a}{a+b}+\frac{b}{a+b}=1$, which is identically true.

Secondly. By hypothesis, the equation of the hyperbola $\frac{x^{2}}{\mathrm{~A}^{2}}-\frac{y^{2}}{\mathrm{~B}^{2}}=1$, is satisfied by the values $x=a, y=b$, which gives (1) $\frac{a^{2}}{\mathrm{~A}^{2}}-\frac{b^{2}}{\mathrm{~B}^{2}}=1$, and also by the other values $x^{2}=\frac{a^{3}}{a+b}, y^{2}=\frac{b^{3}}{a+b}$.

which gives (2)

$$
\frac{a^{3}}{(a+b) \mathrm{A}^{2}}-\frac{b^{3}}{(a+b) \mathrm{B}^{2}}=1 \text {. }
$$

Subtracting (2) from (1)

$$
\frac{a^{2} b}{(a+b) \mathrm{A}^{2}}-\frac{b^{2} a}{(a+b) \mathrm{B}^{2}}=0
$$

$\therefore \frac{a}{\mathrm{~A}^{2}}=\frac{b}{\mathrm{~B}^{2}}$ which gives the remarkable result $\mathrm{A}^{2}: \mathrm{B}^{2}:: a: b$, showing that the elliptic semi-axes are in the duplicate ratio of the hyperbolic ones. Hence if $\mathrm{A}^{2}=k a, \mathrm{~B}^{2}=k b$ ( $k$ being an indeterminate. $)$

To determine its value, we resume the equation (1), $\frac{a^{2}}{\mathrm{~A}^{2}}-\frac{b^{2}}{\mathrm{~B}^{2}}=1$ which gives $\frac{a^{2}}{k a}-\frac{b^{2}}{k b}=1$, whence $k=a-b$.

Therefore the squares of the semi-axes of the hyperbola, are $A^{2}=a(a-b)$, $\mathrm{B}^{2}=b(a-b)$. From whence, by addition, $\mathbf{A}^{2}+\mathrm{B}^{2}=a^{2}-b^{2}$.

It remains now to verify the confocality of the two curves. If $\mathrm{C}$ is their common centre, and $\mathrm{F}$ the focus of the ellipse, we have $\mathrm{CF}^{2}=a^{2}-b^{2}$, by the property of the ellipse, and if $\mathrm{F}^{\prime}$ is the focus of the hyperbola, we have $\mathrm{CF}^{\prime 2}=\mathrm{A}^{2}+\mathrm{B}^{2}$ by the property of the hyperbola. $\therefore$ if $F$ and $\mathrm{F}^{\prime}$ are the same point

$$
\mathrm{CF}^{2}=\mathrm{CF}^{2} \text { or } a^{2}-b^{2}=\mathrm{A}^{2}+\mathrm{B}^{2} .
$$

But we have shown that this equation exists, and therefore the curves are confocal.

Thus we have proved that the squares of the

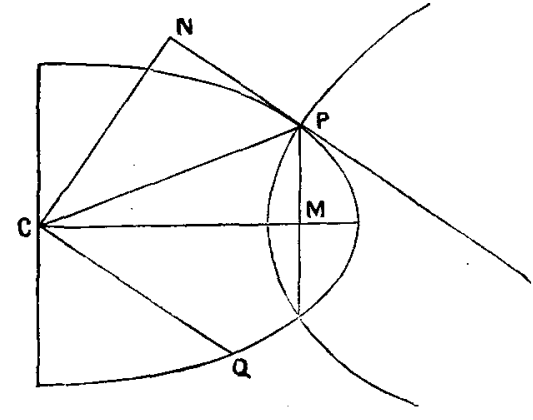

Fig. 9. co-ordinates of the point $\mathbf{P}$, where the confocal ellipse and hyperbola intersect, are,-

$$
x^{2}=\frac{a^{3}}{a+b}, y^{2}=\frac{b^{3}}{a+b} \text {. }
$$

Therefore (1) $\mathrm{CP}^{2}=x^{2}+y^{2}=\frac{a^{3}+b^{3}}{a+b}=a^{2}-a b+b^{2}$

Let CQ be the conjugate to CP.

$\therefore$ (2) $\mathrm{CP}^{2}+\mathrm{CQ}^{2}=a^{2}+b^{2}$ by a property of the ellipse.

Subtracting (1) from (2), we find $\mathrm{CQ}^{2}=a b$.

Let $\mathrm{CN}$ be perpendicular on the tangent $\mathrm{PN}$, then by another property of the 
ellipse, $\mathrm{CN} . \mathrm{CQ}=a b$; whence $\mathrm{CN}^{2} . \mathrm{CQ}^{2}=a^{2} b^{2}$. Divide this equation by $\mathrm{CQ}^{2}=a b$ $\therefore \mathrm{CN}^{2}=a b$, and $\therefore \mathrm{CN}=\mathrm{CQ}$. But now, since $\mathrm{CP}^{2}=a^{2}+b^{2}-a b$, and $\mathrm{CN}^{2}=a b$, therefore $\mathrm{PN}^{2}=\mathrm{CP}^{2}-\mathrm{CN}^{2}=a^{2}+b^{2}-2 a b, \therefore \mathrm{PN}=a-b$, which is the theorem we undertook to demonstrate.**

It is curious that CQ when prolonged, becomes the asymptote of the hyperbola. Perhaps we have already offered sufficient proof of this, but the reader may not object to see it proved in another manner.

The equation to the ellipse $\frac{x^{2}}{a^{2}}+\frac{y^{2}}{b^{2}}=1$ gives $\frac{d y}{d x}=-\frac{x}{y} \cdot \frac{b^{2}}{a^{2}}$. But the value of $\frac{x}{y}$ at FAGNaNI's point is $\frac{a^{\frac{3}{2}}}{b^{\frac{3}{2}}}=\frac{A^{3}}{\bar{B}^{3}}$. Therefore $\frac{d y}{d x}=-\sqrt{\frac{b}{a}}=-\frac{\mathrm{B}}{\mathrm{A}}$. But by the property of the hyperbola, if $\mathrm{C}$ be the centre and $\mathrm{CA}, \mathrm{CB}$ (or $\mathrm{A}, \mathrm{B}$ ), the semi-axes, the asymptote $C D$ will be found by completing the rectangle ACBD, and joining CD. $\therefore$ the asymptote makes, with the axis $\mathrm{CA}$ an angle, whose tangent $=\frac{\mathrm{DA}}{\mathrm{CA}}=\frac{\mathrm{B}}{\mathrm{A}}$, and the other asymptote makes an equal angle below the axis,

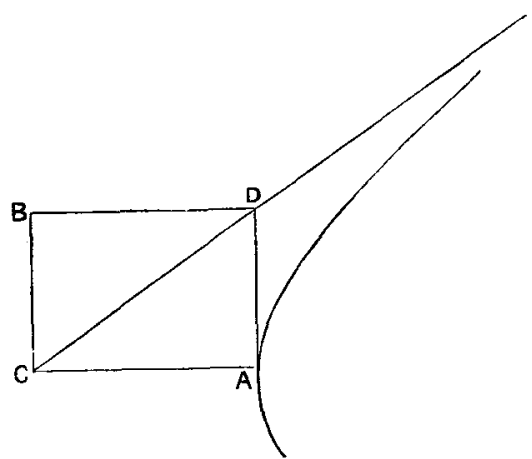

Fig. 10. whose tangent is therefore $-\frac{\mathrm{B}}{\mathrm{A}}$. But we found $\frac{d y}{d x}=-\frac{\mathrm{B}}{\mathrm{A}}$; therefore, the tangent to the ellipse at the point $\mathrm{P}$, or $(x, y)$ is parallel to the second asymptote. Consequently, the conjugate semi-diameter $C Q$, is a portion of that asymptote.

Since the two curves are confocal, they ought to intersect at right angles.

Let us verify this. We have seen that the equation to the ellipse gives $\frac{d y}{d x}=-\frac{\mathrm{B}}{\mathrm{A}}$ at FAGN ANI's point. But the equation to the hyperbola $\frac{x^{2}}{\mathrm{~A}^{2}}-\frac{y^{2}}{\mathrm{~B}^{2}}=1$ gives at the same point $\frac{d y}{d x}=\frac{x}{y} \cdot \frac{\mathrm{B}^{2}}{\mathrm{~A}^{2}}=\frac{\mathrm{A}^{3}}{\overline{\mathrm{B}}^{3}} \cdot \frac{\mathrm{B}^{2}}{\overline{\mathrm{A}}^{2}}=\frac{\mathrm{A}}{\mathrm{B}}$. But these two results, $\frac{\mathrm{B}}{\mathrm{A}}$ in the ellipse, and $\frac{A}{B}$ in the hyperbola (neglecting the signs) are reciprocals. Therefore at the point of intersection the two curves make angles with the axis, whose tangents are reciprocals, and therefore they intersect at right angles.

Hence this curious theorem "At the point of intersection P, the normal to the hyperbola is parallel to one of its asymptotes."

* These properties, viz., that $\mathrm{CN}=\mathrm{CQ}=\sqrt{a \bar{b}}$, and that $\mathrm{PN}=a-b$ are proved by BRINkLEY in quite a different manner (Trans. of the Royal Irish Academy, vol. ix. p. 146, \&c.). He likewise proves, that if $\mathrm{CQ}$ produced cuts in $\mathrm{O}$, the circle described on the axis major as diameter, a perpendicular let fall from $O$ on the axis, cuts the ellipse in FaGNan's point. But $I$ have shown that $C Q$ produced is the asymptote of the hyperbola, $\therefore$ an ordinate to the ellipse at FaGNan's point, passes through the intersection of the asymptote and circle. In other words, the common chord of the ellipse and hyperbola, being produced, becomes the common chord of the circle and the two asymptotes. 
For that normal is tangent to the ellipse, and the asymptote coincides with the conjugate diameter.

Now, let us draw three vectors to the hyperbola, from the centre, making the angles $\theta, \theta^{\prime}, \theta^{\prime \prime}$, respectively with the axis. The first to be drawn to a point infinitely distant (and, therefore, it will be the asymptote), the second to the point $\mathrm{D}$, and the third to the point $\mathrm{P}$ (see fig. 8). Then, if we call $\tan \theta=t$, we have the following curious property,-

$$
\begin{aligned}
& \tan \theta^{\prime}=t^{2} \\
& \tan \theta^{\prime \prime}=t^{3}
\end{aligned}
$$

For $\tan \theta=\frac{\mathrm{B}}{\mathrm{A}}$, and $\tan \theta^{\prime}=\frac{b}{a}=\frac{\mathrm{B}^{2}}{\mathrm{~A}^{2}}$ and $\tan \theta^{\prime \prime}=\frac{y}{x}$, at the point where the curves intersect; and $\therefore=\sqrt{ } \frac{\overline{b^{3}}}{a^{3}}=\frac{B^{3}}{\mathrm{~A}^{3}}$.

Since $\theta$ is less than $45^{\circ}$, and $\therefore \tan \theta$ less than 1 , the angles $\theta, \theta^{\prime}, \theta^{\prime \prime}$, become successively smaller.

Another remarkable property is the following (see fig. 9) :-At the point $P$, where the curves intersect, and where the elliptic quadrant is algebraically divided according to FAGNaNI's theorem, the line PN intercepted by the perpendicular $\mathrm{CN}$ on the tangent, is a maximum. For if we examine in any ellipse what must be the position of the point $\mathrm{P}$, in order that PN may be a maximum, we shall find it to be characterized by those values which we have already shown to belong to $\mathbf{P}$ in FAGNani's theorem. This may be shown as follows :-

In any ellipse, let $\mathrm{C}$ be the centre, $\mathrm{CP}, \mathrm{CQ}$, conjugate diameters, $\mathrm{PN}$ a tangent, and $\mathrm{CN}$ or $p$ the perpendicular to it.

We have

$$
\mathrm{CQ}=\frac{a b}{p} \therefore \mathrm{CQ}^{2}=\frac{a^{2} b^{2}}{p^{2}}
$$

But

$$
\mathrm{CP}^{2}=a^{2}+b^{2}-\mathrm{CQ}^{2}=a^{2}+b^{2}-\frac{a^{2} b^{2}}{p^{2}} \text {. }
$$

Subtract

$$
\mathrm{CN}^{2}=p^{2}
$$

$$
\therefore \mathrm{PN}^{2}=a^{2}+b^{2}-\frac{a^{2} b^{2}}{p^{2}}-p^{2} \text {. }
$$

Let this be a maximum. Therefore since $a^{2}+b^{2}$ is constant, $\frac{a^{2} b^{2}}{p^{2}}+p^{2}$, must be a minimum, or putting $a^{2} b^{2}=n$, and $p^{2}=x$, then $\frac{n}{x}+x$, is a minimum.

Differentiating this, we find $x=\sqrt{n}$; or $p^{2}=a b$. From this value we find $\mathrm{CQ}^{2}=a b$, and $\therefore \mathrm{CQ}=p$, and $\mathrm{PN}^{2}=a^{2}+b^{2}-a b-a b ; \therefore \mathbf{P N}=a-b$. And as these were the values which we found before for the same lines, it is evident that $P$ is the same point which we were considering before. Therefore, at the point $\mathbf{P}$, which may be called FAGNanI's point, the line PN is a maximum. 
Additional Remarks.

I will here add two or three other theorems, which have suggested themselves in the course of this inquiry.

If $\mathrm{BCA}$ is an ellipse, and $\mathbf{P}$ is Fagnani's point, and the tangent OPT is drawn terminated by the axes produced, then $\mathrm{OP}=\mathrm{CA}$, and $\mathrm{PT}=\mathrm{CB}$.

Demonstration.-Let $\mathrm{CA}=a, \mathrm{CB}=b, \mathrm{CN}=x$, $\mathrm{PN}=y$. The equation is $\frac{x^{2}}{a^{2}}+\frac{y^{2}}{b^{2}}=1$. At FAGNanI's point we have,

$$
x^{2}=\frac{a^{3}}{a+b} \text { and } y^{2}=\frac{b^{3}}{a+b} .
$$

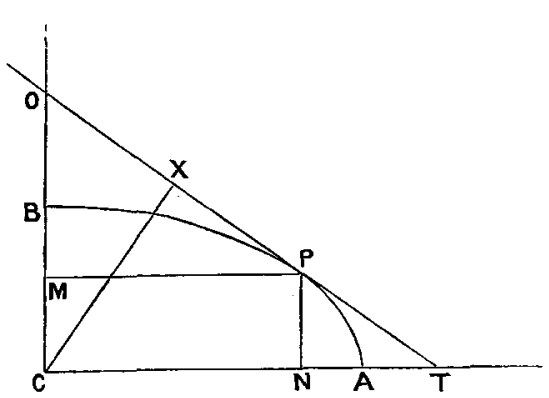

Fig. 11.

Therefore $a+b=\frac{a^{3}}{x^{2}}=\frac{b^{3}}{y^{2}}$. Hence $a(a+b)=\frac{a^{4}}{x^{2}}$ and $b(a+b)=\frac{b^{4}}{y^{2}}$.

Whence by addition $(a+b)^{2}=\frac{a^{4}}{x^{2}}+\frac{b^{4}}{y^{2}}$.

Now we have by a general property of the ellipse $\mathrm{CT}=\frac{a^{2}}{x}$ and $\mathrm{OC}=\frac{b^{2}}{y}$, whence $\mathrm{OT}^{2}=\frac{a^{4}}{x^{2}}+\frac{b^{4}}{y^{2}}$, which we have just proved to be equal to $(a+b)^{2}$. Therefore we have the curious result, $\mathrm{OT}=a+b$.

Now,

$$
\text { OT : OP : : CT : MP; }
$$

or,

$$
a+b: \mathrm{OP}:: \frac{a^{2}}{x}: x \text {. }
$$

Therefore $\mathrm{OP}=(a+b) a^{a^{2}}$. And since at F $x_{\mathrm{AGNANI}}{ }^{2}$ s point $\frac{x^{2}}{a^{2}}=\frac{a}{a+b}$, it follows that $\mathrm{OP}=a$. And similarly it is shown that $\mathrm{PT}=b$.

From whence the following curious theorem follows,-It is well known that if OCT is a right angle, and OT is a line of given length, which moves so as to keep its extremities constantly in the lines CO, CT, then any point $P$ of the line OT will generate an ellipse. But there is only one position of the line OT in which it touches the ellipse.

Theorem.-If the generating line OT touches the ellipse, the point of contact $P$ is Fagnani's point.

Hence, if we let fall the perpendicular $C X$ upon $O T$, then $O X=P T=$ semi-axis minor. For $\mathrm{OP}=a$, and we have seen in the course of this investigation, that

$$
\mathrm{PX}=a-b \therefore \mathrm{OX}=\mathrm{OP}-\mathrm{PX}=b \text {. }
$$

The following corollary is also worth remarking.

$$
\mathrm{OP}-\operatorname{arc} \mathrm{BP}=\mathrm{PT}-\operatorname{arc} \mathrm{PA} \text {. }
$$

VOL. XXIII. PART II. 
And also the following:-Of all the lines, which touch the ellipse, and are terminated by the axes produced, the shortest is OT, which touches at FAGNANI's point.

The simplest proof of this, is by the doctrine of infinitesimals. Let the line of constant length OT (which we called above the generating line) assume another position $0^{\prime} T^{\prime}$, it will now be a secant to the ellipse, and $\mathbf{P}$ will occupy another point in the curve. But if the position $O^{\prime} T^{\prime}$ be taken infinitely near to OT, and $\mathbf{P}^{\prime}$ to $\mathbf{P}$, then $\mathrm{O}^{\prime} \mathrm{T}^{\prime}$ must be considered as still being a tangent; and thus we see that the tangent at $\mathbf{P}^{\prime}$, limited by the axes produced, has the same length whicb it had before [the part $O P$ having gained an infinitesimal quantity $\delta$, and the part PT having lost exactly the same], which is the character of a minimum. Therefore, OT is the minimum tangent, terminated by the axes produced. It is curious, that while $\mathrm{OT}=a+b$ is the Minimum of its kind, $\mathrm{PX}=a-b$ is the Maximum of its kind, as we proved in another part of this Memoir.

This result may also be obtained by the differential calculus, as thus :-

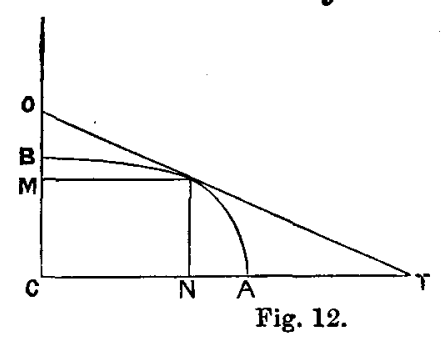

$$
\begin{aligned}
& \mathrm{CT}=\frac{\mathrm{CA}^{2}}{\mathrm{CN}}=\frac{a^{2}}{x} \\
& \mathrm{OC}=\frac{\mathrm{CB}^{2}}{\mathrm{CM}}=\frac{b^{2}}{y} \\
& \therefore \mathrm{OT}^{2}=\frac{a^{4}}{x^{2}}+\frac{b^{4}}{y^{2}}
\end{aligned}
$$

And when OT is a minimum,

$$
\frac{a^{4} d x}{x^{3}}=-\frac{b^{4} d y}{y^{9}}
$$

But by differentiating the equation to the curve, $\frac{x^{2}}{a^{2}}+\frac{y^{2}}{b^{2}}=1$, we find $\frac{x d x}{a^{2}}=-\frac{y d y}{b^{2}}$, which is always true.

Dividing the first of these equations by the second, we get $\frac{a^{6}}{x^{4}}=\frac{b^{6}}{y^{4}}$ whence $\frac{x^{2}}{y^{2}}=$ $\frac{a^{3}}{b^{3}}$, which is the property which characterises FAGNANI's point.

I will terminate this paper, by giving some remarkable properties of confocal ellipses and hyperbolas.

Lemma.-Let XY be the directrix of an ellipse, and $\mathrm{P}$ any point, we have by a property of the ellipse

$$
\begin{aligned}
& \mathrm{HP}: \mathrm{PX}:: e: 1 \\
& e \text { being the excentricity, } \\
\therefore & \mathrm{PX}=\frac{\mathrm{HP}}{e}
\end{aligned}
$$


Therefore, calling $\mathrm{CN}, x$, we have, when the point is at the extremity of the minor axis,

$$
\begin{aligned}
e: 1 & :: \mathrm{HB}: \mathrm{BO} \\
& : \mathrm{HB}: \mathrm{CN}+\mathrm{PX} \\
& :: a: x+\frac{\mathrm{HP}}{e}
\end{aligned}
$$

Therefore $\quad a=e x+\mathrm{HP}$

and $\mathrm{HP}=a-e x$.

This equation to the ellipse may often be useful.

Theorem A.-If an ellipse and hyperbola are confocal, the line from the focus

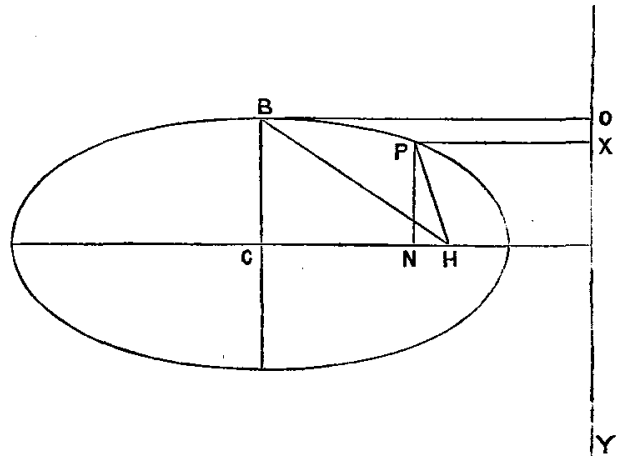

Fig. 13. to the point of intersection equals the distance between the Vertices. Let $\mathrm{S}, \mathrm{H}$, be the foci, $\mathbf{P}$ the point of intersection, $a, b$, the semi-axes of the ellipse; A, B, those of the hyperbola, and V its Vertex,

$\mathrm{SP}+\mathrm{HP}=2 \mathrm{CA}$, and $\mathrm{SP}-\mathrm{HP}=2 \mathrm{CV}$

$\because 2 \mathrm{HP}=2(\mathrm{CA}-\mathrm{CV})=2 \mathrm{VA}$

$\therefore \quad \mathrm{HP}=\mathrm{VA}=a-\mathrm{A}$.

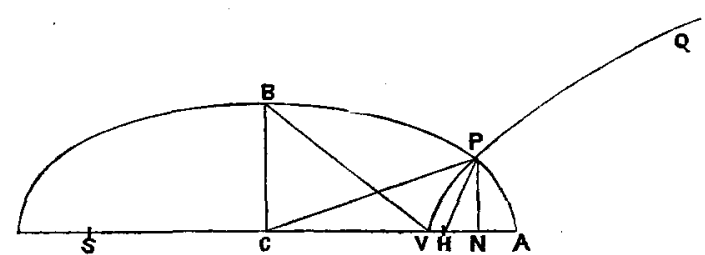

Fig. 14.

The distance $\mathrm{CH}$, between the centre and focus is usually expressed by ae, or $e$ times the semi-axis major. But since in theorems concerning two or more confocal conics, $\mathrm{CH}$ is the only invariable line, it is convenient to denote it by unity. We will therefore in the sequel suppose $\mathrm{CH}$, or $a e=1$; and, therefore $e=\frac{1}{a}$. It must be borne in mind that the condition of confocality gives the following relation between the axes,-

$$
a^{2}-b^{2}=1=\mathrm{A}^{2}+\mathrm{B}^{2} .
$$

Theorem B.-The same suppositions being made as in the last theorem, the co-ordinates of the point of intersection have the values

$$
x=\mathrm{A} a \quad y=\mathrm{B} b .
$$

And CP the central distance of the point of intersection, is equal to VB, which also has the value,-

$$
\sqrt{A^{2}+a^{2}-1} \text {. }
$$

For we proved in the last theorem that

$$
\begin{aligned}
& \mathrm{HP}=a-\mathrm{A} \text {, but by the Lemma (putting } e=\frac{1}{a} \text { ), } \\
& \mathrm{HP}=a-\frac{x}{a}
\end{aligned}
$$

Therefore,

$$
\frac{x}{a}=\mathrm{A}, \text { or } x=\mathrm{A} a \text {. }
$$

It remains to find the value of $y$. 
Take the equation to the ellipse $\frac{x^{2}}{a^{2}}+\frac{y^{2}}{b^{2}}=1$, and substitute for $x$ its value $A a$,

$$
\therefore \mathrm{A}^{2}+\frac{y^{2}}{b^{2}}=1, \therefore \frac{y^{2}}{b^{2}}=1-\mathrm{A}^{2}=\mathrm{B}^{2}, \therefore y=\mathrm{B} b \text {. }
$$

And a similar result is obtained from the equation to the hyperbola, viz.-

$$
\frac{x^{2}}{\mathrm{~A}^{2}}-\frac{y^{2}}{\mathrm{~B}^{2}}=1 \text {. }
$$

For this gives, by putting for $x$ its value $\mathrm{A} a$,

$$
a^{2}-\frac{y^{2}}{\mathbf{B}^{2}}=1, \therefore \frac{y^{2}}{\mathbf{B}^{2}}=a^{2}-1=b^{2} \quad \therefore y=\mathrm{B} b \text {. }
$$

The remainder of the theorem is thus demonstrated,-

Since

$$
\begin{aligned}
& x=\mathrm{A} a, \text { and } y=\mathrm{B} b, \\
& \mathrm{CP}^{2}=x^{2}+y^{2}=\mathrm{A}^{2} a^{2}+\mathrm{B}^{2} b^{2} \\
& =\mathrm{A}^{2} a^{2}+\left(1-\mathrm{A}^{2}\right)\left(a^{2}-1\right)=\mathrm{A}^{2}+a^{2}-1=\mathrm{A}^{2}+b^{2} \\
& =\mathrm{VB}^{2} ; \text { and therefore } \mathrm{CP}=\mathrm{VB} .
\end{aligned}
$$

From hence we derive the following remarkable property of confocal conics:If two ellipses and two hyperbolas have all of them the same foci, and inter-

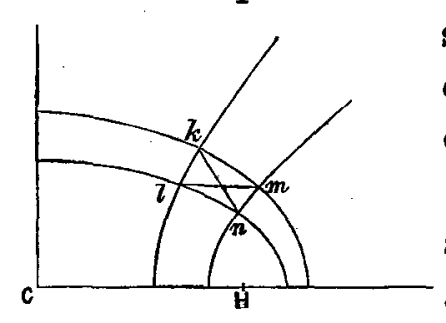

Fig. 15. sect in four points, $k, l, m, n$, forming a curvilinear quadrilateral, the straight lines $l m, k n$, which form the diagonals of this quadrilateral, are equal to each other.

Demonstration.-Let the semi-axes of the ellipse and hyperbola nearest the centre be called $a, b$, and $\mathrm{A}, \mathrm{B}$; and let those of the ellipse and hyperbola farthest from the centre be called $a^{\prime}, b^{\prime}$ and $\mathrm{A}^{\prime}, \mathrm{B}^{\prime}$.

Let the co-ordinates of the point $\quad l$ be $x$ and $y$.

$$
\begin{aligned}
& \text { those of } \quad m \ldots x_{1} \ldots y_{1} \\
& \text { those of } \quad k \ldots x_{2} \ldots y_{2} \\
& \text { those of } \quad n \ldots x_{8} \ldots y_{3} \text {. }
\end{aligned}
$$

Then the square of the diagonal

$$
\begin{aligned}
& l m=\left(x_{1}-x\right)^{2}+\left(y_{1}-y\right)^{2} \\
& k n=\left(x_{3}-x_{2}\right)^{2}+\left(y_{3}-y_{2}\right)^{2}
\end{aligned}
$$

And of the diagonal

What we have to prove, therefore, is that these two expressions are equal.

Now, since the point $l$ belongs to the ellipse whose semi-axes are $a, b$, and also to the hyperbola whose semi-axes are $A, B$, we have

Therefore,

$$
\begin{aligned}
& x=\mathrm{A} a \quad y=\mathrm{B} b \text {. And for similar reasons, } \\
& x_{1}=\mathrm{A}^{\prime} a^{\prime} \quad y_{1}=\mathrm{B}^{\prime} b^{\prime} \\
& x_{2}=\mathrm{A} a^{\prime} \quad y_{2}=\mathrm{B} b^{\prime} \\
& x_{3}=\mathrm{A}^{\prime} \quad a y_{3}=\mathrm{B}^{\prime} b \\
& \text { And } \quad x_{2} x_{8}=\mathrm{A} a^{\prime} \times \mathrm{A}^{\prime} a \text {; }
\end{aligned}
$$

And therefore, $x x_{1}=x_{2} x_{3}$, each side being the product of the four semi-axes major. 
In a similar way it is shown that $y y_{1}=y_{2} y_{3}$, each side being the product of the four semi-axes minor.

Now, we wish to prove the equality of the expressions

$$
\left(x_{1}-x\right)^{2}+\left(y_{1}-y\right)^{2} \text { and }\left(x_{3}-x_{2}\right)^{2}+\left(y_{3}-y_{2}\right)^{2}
$$

Subtract from them respectively the quantities

$$
-2\left(x x_{1}+y y_{1}\right) \text { and }-2\left(x_{3} x_{2}+y_{3} y_{2}\right)
$$

which we have just proved to be equal.

The remainders will be

$$
\begin{gathered}
x^{2}+x_{1}{ }^{2}+y_{1}{ }^{2}+y^{2}, \\
x_{2}{ }^{2}+x_{3}{ }^{2}+y_{2}{ }^{2}+y_{3}{ }^{2} .
\end{gathered}
$$$$
\text { And }
$$

And now it is required to prove that these two remainders are equal.

But we have proved in theorem $B$, that

$$
\begin{aligned}
x^{2}+y^{2} & =\mathrm{A}^{2}+a^{2}-1 ; \text { and by similar reasoning, } \\
x_{1}{ }^{2}+y_{1}{ }^{2} & =\mathrm{A}^{\prime 2}+a^{\prime 2}-1 .
\end{aligned}
$$

Therefore $\left(x^{2}+y^{2}\right)+\left(x_{1}^{2}+y_{1}^{2}\right)=-2+$ the sum of the squares of the 4 semi-axes major.

And by similar reasoning, $\left(x_{2}{ }^{2}+y_{2}{ }^{2}\right)+\left(x_{3}{ }^{2}+y_{3}{ }^{2}\right)$ is equal to the same quantity. Therefore the two remainders are equal; and therefore the theorem is demonstrated.

From this theorem several others may be deduced, by giving extreme values to the four curves.

In the first place, if the two ellipses are drawn infinitely near to each other, and likewise the two hyperbolas infinitely near to each other, then, because confocal conics always intersect at right angles, the small quadrilateral formed by the four intersections will be a rectangle, and of course its diagonals will be equal.

Next suppose that the axis minor of the first ellipse is infinitely diminished, the quadrant of the curve will be reduced to the straight line $\mathrm{CH}$, extending from the centre to the focus. At the same time let the semi-axis major of the first hyperbola be infinitely diminished, and the vertex of it will then coincide with the centre, and the curve itself will become a straight line in the direction of the axis minor produced to infinity. The four curves will thus be reduced to one ellipse and one hyperbola, and two rectangular straight lines. The quadrilateral figure then becomes BCVP (see figure 14), and our theorem asserts that in that case $\mathrm{CP}=\mathrm{BV}$, the truth of which was independently proved in theorem $\mathrm{B}$.

Now, let the other hyperbola also have its axis minor infinitely diminished, its vertex will then coincide with the focus, and the curve will be reduced to the straight line HA produced to infinity. The line CP then becomes CA, and BV 
becomes $\mathrm{BH}$ (see fig. 14). Our theorem asserts, that in this case $\mathrm{CA}=\mathrm{BH}$, the truth of which is otherwise manifest.

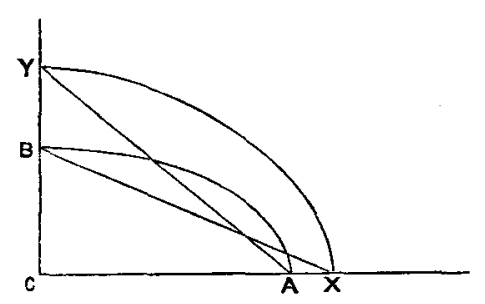

Fig. 16.

Again, let CA, CB, and CX, CY, be the semi-axes of the two confocal ellipses, but let the confocal hyperboles be reduced as before to the straight lines $\mathrm{CBY}$ (produced to infinity), and AX (produced to infinity), the intersections of the first hyperbola with the ellipses will be $B$ and $Y$, those of the second $A$ and $X$. The diagonals will become the lines $\mathrm{BX}$ and $\mathrm{AY}$; and our theorem asserts that in this case $\mathrm{BX}=\mathrm{AY}$, which may be proved independently as follows :-

Let the semi-axes of the smaller ellipse be $a, b$, and of the larger one $a, \beta$.

Since they are confocal, $a^{2}-b^{2}=\alpha^{2}-\beta^{2}$, and therefore

$a^{2}+\beta^{2}=\alpha^{2}+b^{2}$.

But

$a^{2}+\beta^{2}=\mathrm{AY}^{2}$, and $\alpha^{2}+b^{2}=\mathrm{BX}^{2}$.

Therefore $\mathrm{AY}=\mathrm{BX}$.

This theorem may be thus enunciated :-

"If the alternate vertices of two confocal ellipses are joined, the lines joining them are equal."

The co-ordinates of $(x, y)$ the point of intersection of a confocal ellipse and hyperbola, may also, if preferred, be readily deduced from first principles, as follows:-

Given the confocal ellipse and hyperbola whose equations are $\frac{x^{2}}{a^{2}}+\frac{y^{2}}{b^{2}}=1$ and $\frac{x^{2}}{\mathrm{~A}^{2}}-\frac{y^{2}}{\overline{\mathrm{B}}^{2}}=1$, with the condition that $x^{2}-b^{2}=\mathrm{A}^{2}+\mathrm{B}^{2}=1$ to find the values of $x$ and $y$ the co-ordinates at the point of intersection?

The equations give respectively $y^{2}=b^{2}-\frac{b^{2}}{a^{2}} x^{2}$ and $y^{2}=\frac{\mathrm{B}^{2}}{\mathrm{~A}^{2}} \cdot x^{2}-B^{2}$. Equating these values of $y^{2}$, we find $\mathrm{B}^{2}+b^{2}=x^{2}\left(\frac{\mathrm{B}^{2} a^{2}+b^{2} A^{2}}{A^{2} a^{2}}\right)$. Now, substituting $1-A^{2}$ for $\mathrm{B}^{2}$, and $a^{2}-1$ for $b^{2}$, we find that $\mathrm{B}^{2}+b^{2}=a^{2}-\mathrm{A}^{2}$, which is also equal to $\mathrm{B}^{2} a^{2}+b^{2} \mathrm{~A}^{2}$, and therefore may be omitted on both sides of the equation, which reduces itself to $1=\frac{x^{2}}{\mathrm{~A}^{2} a^{2}}$ whence $x=\mathrm{A} a$.

Additional Note.-In the second page of this Memoir it is said, that the total deviation tends to increase without limit. To this it may be objected, that the successive deviations may possibly form a diminishing series, having a finite sum. But it can be easily shown that two successive deviations, when they are small, are (very nearly) equal to each other. Moreover, after diminishing to a certain extent, the deviations increase again, having one maximum and one minimum value in the course of one entire revolution round the circle. 